\title{
Morfologia externa do adulto de Almeidaia aidae Mielke \& Casagrande (Lepidoptera, Saturniidae, Arsenurinae, Almeidaiini). III. Abdome ${ }^{1}$
}

\author{
Amabílio J. A. de Camargo ${ }^{2}$; Mirna M. Casagrande ${ }^{3}$; Olaf H.H. Mielke ${ }^{3} \&$ Eurides Furtado ${ }^{4}$ \\ ${ }^{1}$ Contribuição número 1541 do Departamento de Zoologia, Universidade Federal do Paraná. \\ ${ }^{2}$ Embrapa Cerrados. Rodovia BR 020, km 18, Caixa Postal 08223, 73310-970 Brasília, Distrito Federal, Brasil. E-mail: \\ amabilio@cpac.embrapa.br \\ ${ }^{3}$ Departamento de Zoologia, Universidade Federal do Paraná. Caixa Postal 19020, 81531-980 Curitiba, Paraná, Brasil. \\ Pesquisadordo CNPq.E-mail:mibras@ufpr.br; omhesp@ufpr.br \\ ${ }^{4}$ Caixa Postal 97, 78400-000 Diamantino, Mato Grosso, Brasil.
}

\begin{abstract}
External morphology of Almeidaia aidae Mielke \& Casagrande (Lepidoptera, Saturniidae, Arsenurinae, Almeidaiini). III. Abdome. The external morphology of adult abdome of Almeidaia aidae Mielke \& Casagrande, 1981 is described and illustrated for the first time. The results obtained were compared with others species of Saturniidae. This species is rare and endemic of the Cerrado region. Biological studies were published by the fourth author. This is the third of a series of papers about the species, the previous two were about head and thorax morphology.
\end{abstract}

KEY WORDS. Brazil; Cerrado region; moth; Neotropical.

RESUMO. A morfologia externa do abdome do adulto de Almeidaia aidae Mielke \& Casagrande, 1981 é descrita e ilustrada pela primeira vez. Os resultados obtidos foram comparados com outras espécies de Saturniidae. É uma espécie rara e endêmica da região do Cerrado. Estudos sobre sua biologia foram publicados pelo quarto autor. Esta é a terceira contribuição sobre a morfologia da espécie tendo sido as anteriores sobre a cabeça e o tórax. PALAVRAS CHAVE. Brasil; mariposa; Neotropical; região do Cerrado.

Almeidaia aidae Mielke \& Casagrande, 1981, era um dos Arsenurinae menos estudados, pouco se conhecendo sobre a sua biologia e morfologia. Recentemente, no entanto, vários exemplares foram coletados e criados em Diamantino, Mato Grosso, Brasil, pelo quarto autor, o que possibilitou o esclarecimento sobre aspectos de sua biologia (FURTADo 2004). Este é o terceiro e último de uma série de três artigos sobre a morfologia externa da espécie, sendo que o primeiro e segundo trataram da morfologia da cabeça e do tórax, respectivamente (CAMARGO et al. 2005a,b). Os caracteres do gênero Almeidaia Travassos, 1937 e espécies componentes são discutidos em Travassos (1937), Gagárin (1966), Lemaire (1980), Mielke \& Casagrande (1981) e CAmargo et al. (2005a,b).

\section{MATERIAL E MÉTODOS}

A metodologia seguida foi similar às dos estudos da cabeça e do tórax (CAMARGo et al. 2005a,b), tanto para a nomenclatura empregada quanto para obtenção e processamento do material. Os exemplares utilizados nesse estudo são provenientes de criações realizadas pelo quarto autor em Diamantino, Mato Grosso.
A escala utilizada em cada ilustração representa $1 \mathrm{~mm}$, salvo indicação na própria figura.

Adaptou-se o tempo de fervura, corante e solução para limpeza, a partir de técnicas usuais para microlepidópteros descritos em Hogue (1963) e Robinson (1976). Apesar dos corantes serem utilizados na preparação de genitálias, este método se mostrou vantajoso também para outras estruturas morfológicas em Saturniidae.

O abdome foi imerso em uma solução de $\mathrm{KOH} 10 \%$ (hidróxido de potássio) e fervido em banho-maria durante 15 minutos para os machos e 10 minutos para as fêmeas. Este diferencial no tempo de fervura deve-se à necessidade de preservação das estruturas, pois nas fêmeas a bolsa copuladora pode ser danificada se o tempo for excessivo. Após a fervura as peças foram limpas com solução desengordurante contendo 50\% de acetona, 30\% de água destilada e 20\% de álcool 90\%. A genitália foi removida e corada durante cinco minutos com mercúrio cromo 1\% diluído em água para os machos e para as fêmeas, o corante usado foi clorasol negro 1\% diluído em álcool 70\% durante três minutos, facilitando assim a visualização de áreas pouco esclerotinizadas. O uso do clorasol mostrou-se vantajo- 

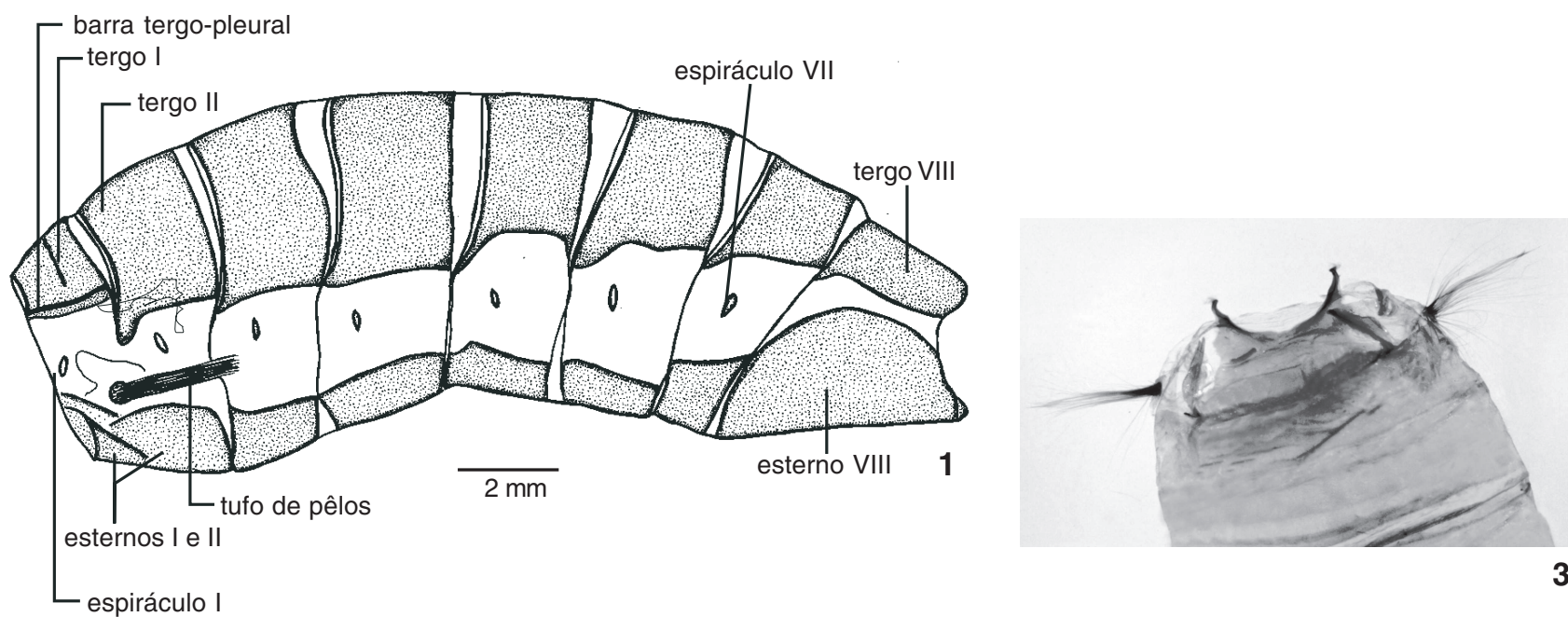

barra tergo-pleural
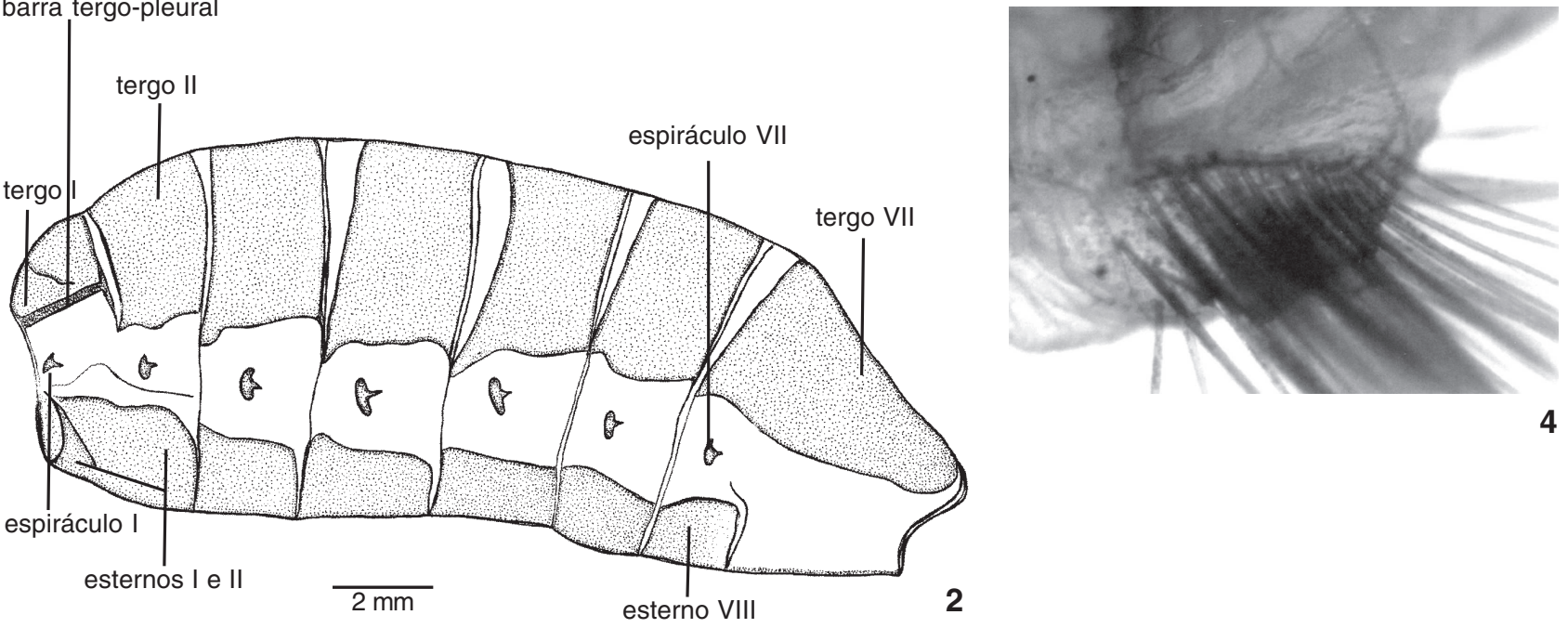

Figuras 1-4. (1) Vista lateral do abdome do macho, genitália removida; (2) vista lateral do abdome da fêmea, genitália removida; (3-4) abdome do macho, tufo de pelos: (3) vista dorsal; (4) detalhe em vista lateral.

so para destacar áreas membranosas como a bolsa copuladora, mas não para peças fortemente esclerotinizadas como acontece com a genitália do macho, pois estas áreas podem colorir em excesso. O excesso de mercúrio pode ser facilmente removido, o que não acontece com o clorasol.

Depois de decorrido o tempo de coloração, o excesso de corante foi removido com a solução desengordurante descrita acima, durante cinco minutos. As ilustrações foram feitas com auxílio de microscópio estereoscópico com câmara clara acoplada. Para a conservação das estruturas foi utilizado GFA, líquido preparado conforme descrito em CASAGRANDE (1979).

As estruturas foram descritas com base na literatura específica para Saturniidae (Michener 1952, FERGUSON 1971, LeMAIRE 1971). Em alguns casos utilizou-se Beutelspacher (1967), Matsuda (1976), Niculescu (1978), Bilotta (1995), Bizarro et al. (2003).

\section{RESULTADOS E DISCUSSÃO}

A morfologia de Almeidaia aidae foi comparada, em seus aspectos gerais, com Eacles imperialis (Drury, 1773) (Ceratocampinae) Michener (1952) e com Automeris postalbida Schaus, 1900 (Hemileucinae) Lemaire (1971).

\section{Abdome (Figs 1-4)}

Formado por dez segmentos distintos, sendo que os dois e três últimos, nos machos e fêmeas respectivamente, apresentam-se modificados, formando as estruturas genitais. Em ambos os sexos, tergo e esterno I apresentam modificações em relação aos demais escleritos devido à articulação com o tórax. Tergo I com dimensões menores que os demais, apresenta na sua face lateral a barra tergo-pleural, fortemente esclerotinizada na fêmea. Em ambos os sexos os esternos I e II encontram-se

Revista Brasileira de Zoologia 23 (1): 157-161, março 2006 


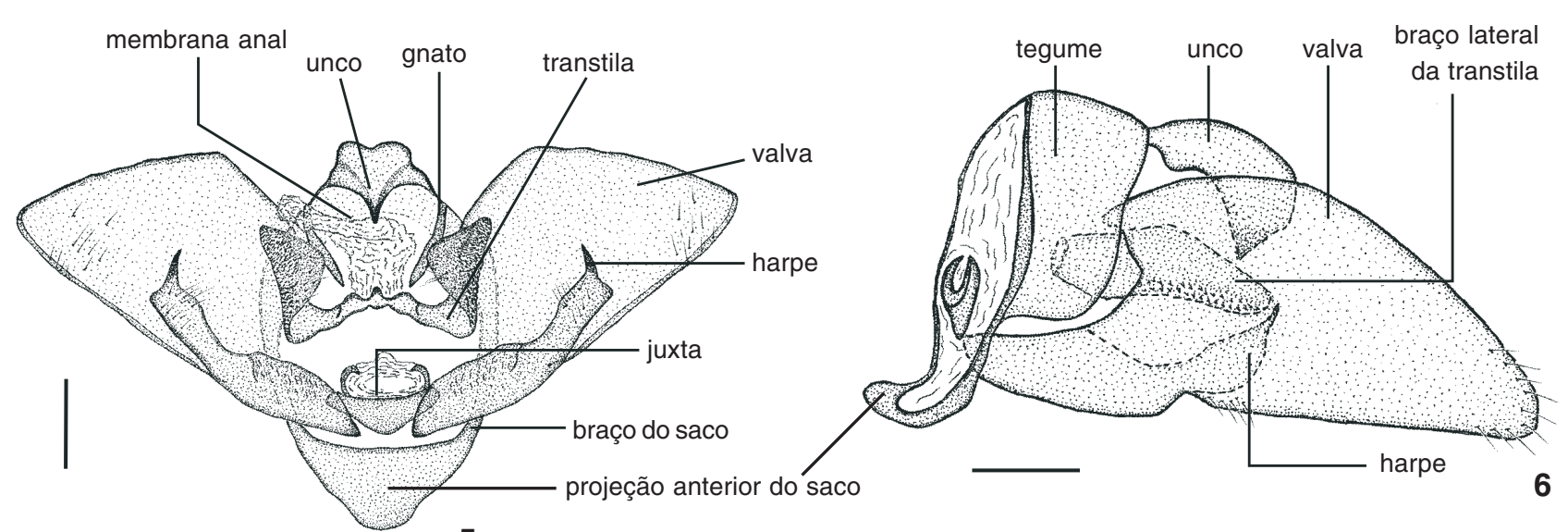

5
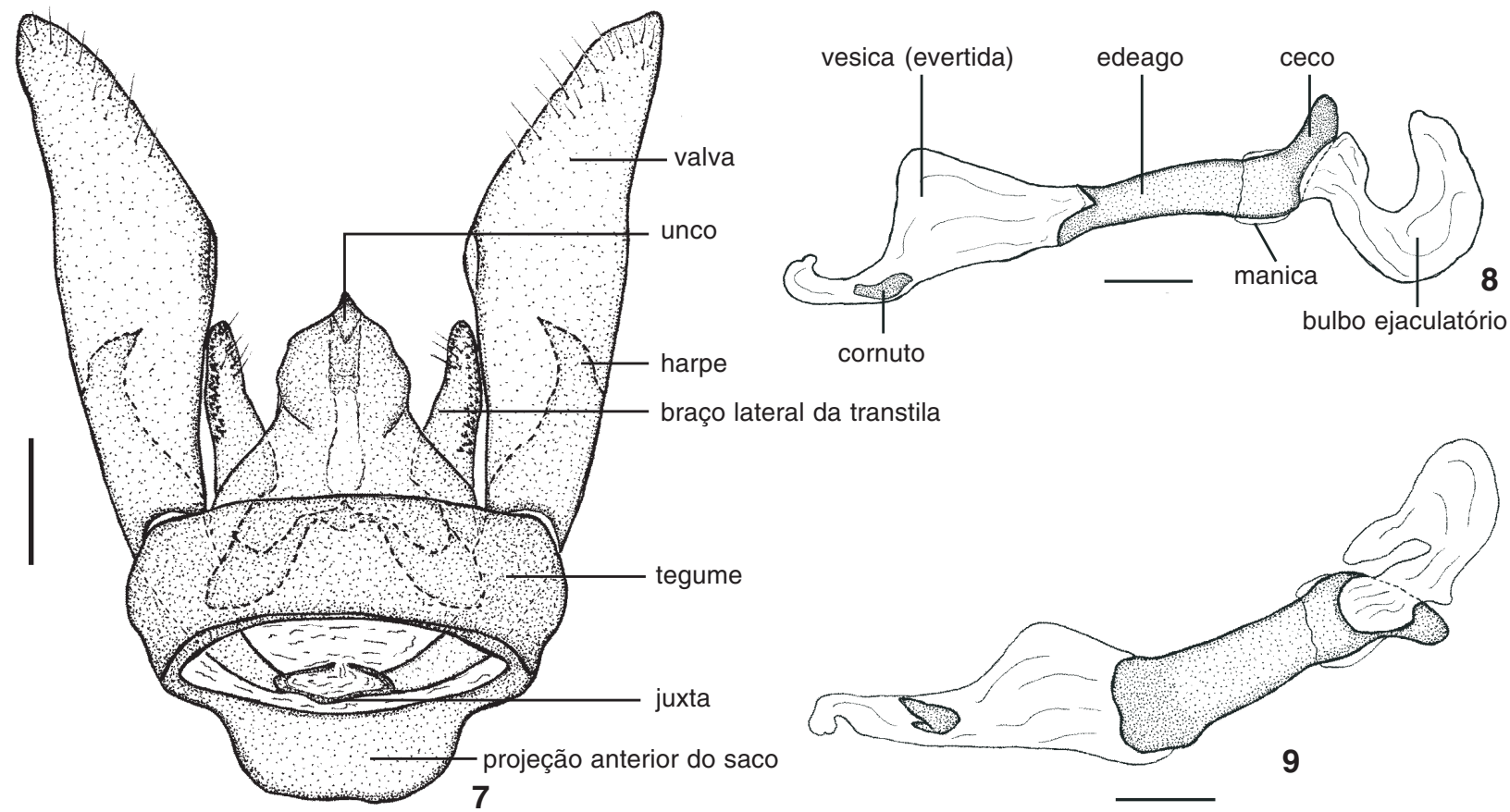

Figuras 5-9. (5-7) Genitália do macho, vista: (5) ventral; (6) lateral; (7) dorsal; (8-9) pênis, vista: (8) lateral; (9) ventral.

praticamente fundidos, sendo de difícil distinção. O tergo VII nas fêmeas apresenta dimensões maiores que nos machos, enquanto que nestes o maior esclerito abdominal é o esterno VIII. A particularidade que se destaca no abdome de $A$. aidae, bem como nos demais Arsenurinae, quando comparados com as demais subfamílias é a presença de um tufo de pelos, de função desconhecida, no primeiro segmento abdominal, inexistente nas demais. Localizados na região central da pleura, nos segmentos de 1 a 7 , os espiráculos em forma de fenda ovalada estão posicionados verticalmente.

\section{Genitália do macho (Figs 5-9)}

As estruturas genitais dos machos das espécies dessa família são muito variáveis, constituindo-se em bons caracteres taxonômicos. O unco pode ser simples, bífido ou trífido; a valva lobada ou simples, com ou sem harpe; o gnato e a transtila podem estar presentes ou ausentes. Mesmo na subfamília Arsenurinae estas variações são acentuadas, portanto não é possível traçar um padrão geral que caracterize o grupo. $\mathrm{O}$ edeago, muitas vezes, é robusto e com vários cornutos, como observado na maioria das espécies de Ceratocampinae, ou apresenta-se como uma estrutura frágil e lisa em outros casos.

Formada a partir da modificação do IX e X segmentos abdominais, consiste basicamente de: tegume, saco, unco, gnato, transtila, juxta, edeago e duas valvas simétricas, que lembram aquelas de E. imperialis por serem largas e curtas. O tegume, uma placa cônica esclerotinizada, se funde ventralmente aos 


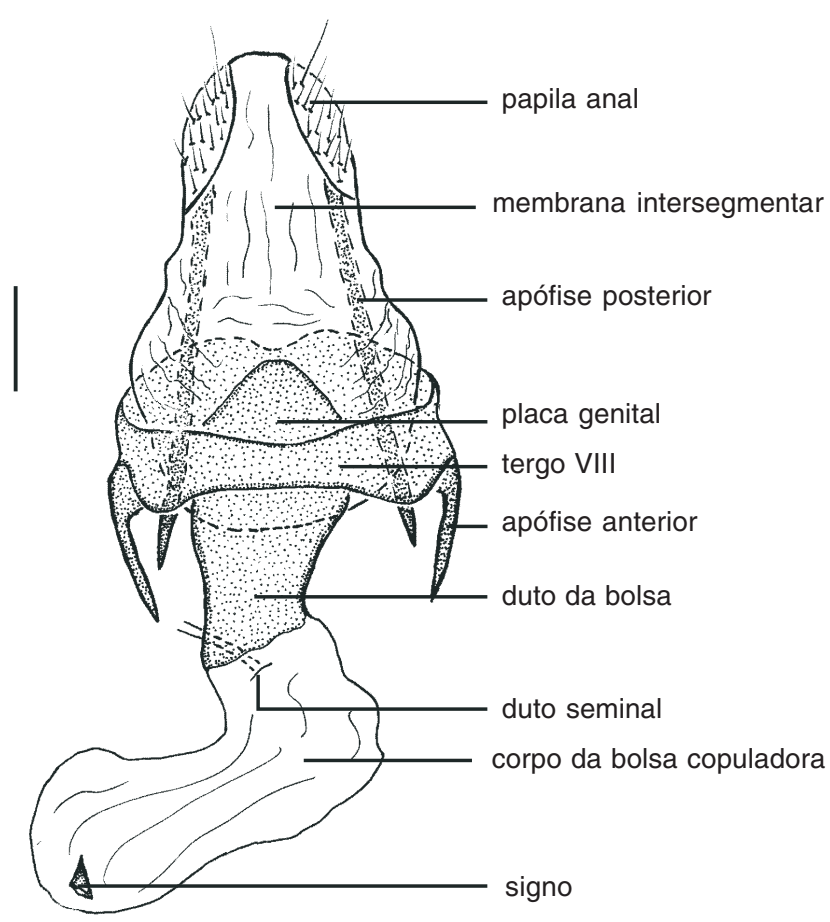

Figura 10. Genitália da fêmea, vista ventral.

braços dorsais do saco. A estrutura mais proximal da genitália do macho é formada pelo nono esterno, o saco, de forma semitriangular, projetada anteriormente e com braços distais que se unem aos braços ventrais do tegume. A projeção anterior do saco em $A$. aidae e $E$. imperialis é curta, isto é, $1 / 3$ do tamanho se comparadas a de $A$. postalbida. Unco, formado pela modificação do tergo $\mathrm{X}$, do tipo simples e como uma projeção distal do tegume, anteriormente largo, afila-se progressivamente até formar uma área estreita puntiforme mais esclerotinizada e dirigida ventralmente, diferente de E. imperialis que é truncado e mais próximo quanto à forma de $A$. postalbida. Em posição látero-ventral, o gnato aparece como um par de projeções laterais, delgadas e fortemente esclerotinizadas que se projetam em direção à área central da transtila. Michener (1952) se refere a estas duas estruturas (gnato e transtila) de forma duvidosa sem identificar cada uma delas com certeza. No entanto, neste caso, o gnato é facilmente distinto da transtila, formando um anel com o unco, conforme descrito por Niculescu (1978). Consideramos a transtila como uma estrutura transversal, entre as bases dorsais das valvas, na parte mediana, fortemente destacada com um par de projeções laterais, largas e rugosas. As valvas são estruturas largas que se projetam ventralmente, dobrando em direção dorsal, formando na base interna o sáculo em cuja porção distal, projeta-se uma estrutura na forma de espinho, a harpe. A juxta é composta de uma placa esclerotinizada semitriangular, entre a base das valvas, em cuja borda interna localiza-se a manica, membrana que sustenta o edeago.

Revista Brasileira de Zoologia 23 (1): 157-161, março 2006
O edeago é formado por um tubo cilíndrico em cuja porção distal aloja a vesica, membranosa com tamanho, quando evertida, aproximadamente igual ao edeago. Distalmente, a vesica apresenta uma estrutura ímpar e esclerotinizada, denominada cornuto. Na porção proximal do edeago, e inferiormente ao ceco - termo utilizado por Lemaire $(1971,1980)$-, está o bulbo ejaculatório de forma semicircular.

\section{Genitália da fêmea (Fig. 10)}

Em Saturniidae a genitália das fêmeas geralmente não contribuem com caracteres taxonômicos, exceto pela presença de uma área esclerotinizada, o signo, ou estrias na bolsa copuladora presentes em algumas espécies. Na subfamília Arsenurinae o signo está presente em $A$. aidae e em quatro das cinco espécies de Copiopteryx Duncan, 1841 (exceto C. derceto), e as estrias na bolsa aparecem somente nessas mesmas quatro espécies de Copiopteryx (Lemaire 1980). Em A. aidae, como nos lepidóteros em geral, a genitália da fêmea é formada a partir da modificação dos três últimos segmentos do abdome. É composta basicamente das papilas anais, formadas a partir dos tergos dos dois últimos segmentos (IX e X); de uma membrana intersegmentar, que dá sustentação às estruturas; dois pares de apófises (anteriores e posteriores) que se projetam para o interior do abdome, onde se fixam os músculos responsáveis pela retração da genitália, pela bolsa copuladora e placa genital.

As papilas anais são recobertas por cerdas e separadas ventralmente por uma área membranosa onde estão localizados o ânus e oviporo. A membrana intersegmentar é longa, recobrindo a maior parte da genitália. As apófises posteriores são mais longas do que as anteriores, e ambas, juntamente com a placa genital, lamelas ante e pós-vaginal, e oitavo tergo são as áreas mais fortemente esclerotinizadas. A bolsa copuladora, com corpo membranoso, liso e sem estrias, apresenta na sua porção proximal um único signo de forma triangular e na porção distal a inserção do duto seminal. O duto da bolsa copuladora ocupa mais de um terço do seu comprimento total e, assim como o óstio da bolsa, apresenta-se fortemente esclerotinizado.

\section{AGRADECIMENTOS}

À Empresa Brasileira de Pesquisa Agropecuária (EMBRAPA), especialmente ao Centro de Pesquisa Agropecuária dos Cerrados (CPAC), que proporcionou ao autor sênior os meios necessários para execução do trabalho. À bióloga Karen Schmidt pelas sugestões. Aos técnicos da Embrapa Cerrados Wellington Cavalcanti, pelo auxílio com o programa de tratamento das figuras, e Jânio Fonseca Silva que providenciou os materiais e equipamentos necessários aos trabalhos de laboratório.

\section{REFERÊNCIAS BIBLIOGRÁFICAS}

Beutelspacher, C. 1967. Estudio morfologico de Erinnys ello (Linnaeus, 1758) (Lepidoptera, Sphingidae). Anales del Instituto de Biología de la Universidad Nacional Autónoma de México, Série Zoología, México, 38 (1): 59-74. 
Bilotta, I. G. 1995. Morfologia comparada do abdome das espécies brasileiras de Morphinae (Lepidoptera, Nymphalidae). Revista Brasileira de Zoologia, Curitiba, 11 (4): 737-748.

BizarRo, J.M.S.; M.M. Casagrande \& O.H.H. MielKe. 2003. Morfologia externa de Thyridia psidii cetoides (Rosenberg \& Talbot) (Lepidoptera, Nymphalidae, Ithomiinae). III. Abdome e apêndices. Revista Brasileira de Zoologia, Curitiba, 20 (4): 681-684.

Camargo, A.J.A. de; M.M. Casagrande; O.H.H. Mielke \& E. FurtaDo. 2005a. Morfologia externa do adulto de Almeidaia aidae Mielke \& Casagrande (Lepidoptera, Saturniidae, Arsenurinae, Almeidaini). I. Cabeça, apêndices e região cervical. Revista Brasileira de Zoologia, Curitiba, 22(3): 619-622.

Camargo, A.J.A. de; M.M. Casagrande; O.H.H. Mielke \& E. FurtaDo. 2005b. Morfologia externa do adulto de Almeidaia aidae Mielke \& Casagrande (Lepidoptera, Saturniidae, Arsenurinae, Almeidaini). II. Tórax e apêndices. Revista Brasileira de Zoologia, Curitiba, 22 (4): 1152-1158.

Casagrande, M.M. 1979. Sobre Caligo beltrao (Illiger).II: Morfologia externa da cabeça do adulto (Lepidoptera, Satyridae, Brassolinae). Revista Brasileira de Biologia, Rio de Janeiro, 39 (1): 223-227.

Fergusson, D.C. 1971. Bombycoidea, Saturniidae (Part). The Moths of North America. London, E.W. Classey and R.B.D. Publications, fascicle 20, 2A, p. 1-153.

FurTado, E. 2004. Almeidaia aidae Mielke \& Casagrande: seus estágios imaturos e notas taxonômicas (Lepidoptera, Saturniidae, Arsenurinae, Almeidaiini) Revista Brasileira de Zoologia, Curitiba, 21 (3): 663 -669.

GaGÁrIn, P. 1966. Sobre o exemplar feminino de "Almeidaia romualdoi" Travassos, 1937. Atas da Sociedade de Biologia do Rio de Janeiro, Rio de Janeiro, 10(4): 95.

Hogue, C.L. 1963. A standard method for mounting whole adult Lepidoptera on slides utilizing polystyrene plastic. Journal of Research on the Lepidoptera, Arcadia, 1 (3): 223-235.

Lemaire, C. 1971. Révision du genre Automeris Hübner et dês genres voisins. Biogéographie, éthologie, morphologie, taxonomie (Lep. Attacidae). Mémoires du Muséum National D'Histoire Naturelle, Paris, 68: 1-576.

Lemaire, C. 1980. Les Attacidae Américains (= Saturniidae). Arsenurinae. Neuilly-sur-Seine, Édition C. Lemaire, 199p.

MatsudA, R. 1976. Morphology and evolution of the insect abdomen. Oxford, Pergamon Press, VIII+534p.

Michener, C.D. 1952. The Saturniidae (Lepidoptera) of the Western Hemisphere, morphology, phylogeny, and classification. Bulletin of the American Museum of Natural History, New York, 98 (5): 335-502.

Mielke, O.H.H \& M.M. Casagrande. 1981. Notas sobre o gênero Almeidaia Travassos, com descrição de uma espécie nova (Lepidoptera, Saturniidae, Arsenurinae). Revista Brasileira de Biologia, Rio de Janeiro, 41 (4): 809-813.

Niculescu, E.V. 1978. L'armure génitale chez les Lépidoptères. Bulletin de La Société Entomologica de Mulhouse (Suppl.): 1-96.

Robinson, G.S. 1976. The preparation of slides of Lepidoptera genitalia with special reference to the microlepidoptera. Entomologists Gazette, Northamptonshire, 27: 127-132.

Travassos, L. 1937. Sobre um novo typo de "Syssphingidae" (Lepidoptera). Rodriguesia, Rio de Janeiro, 10: 199-201.

Recebido em 01.IV.2004; aceito em 17.I.2006. 